\title{
VARIABILIDAD TÉRMICA EN LA REGIÓN NORORIENTAL DE LA PENÍNSULA DE BRUNSWICK, MAGALLANES, CHILE, EMPLEANDO DATOS LANDSAT.
}

\author{
THERMAL VARIABILITY IN THE NORTHEASTERN AREA OF BRUNSWICK \\ PENINSULA, MAGALLANES, CHILE, USING LANDSAT DATA
}

Carlos Olave-Solar ${ }^{1}$, Ariel Santana ${ }^{2}$, Nicolás Butorovic ${ }^{1} \&$ Paola Acuña $^{1}$

\begin{abstract}
RESUMEN
Convencionalmente, los registros de la temperatura superficial (TS) se han obtenido mediante estaciones meteorológicas instaladas en sectores geográficos específicos y es común que estos registros sean extrapolados a extensiones geográficas más amplias. Sin embargo, las temperaturas varían dependiendo de factores locales como la topografía y la latitud, por tanto es esperable que varíen dentro de un área, en un mismo momento. Estas variaciones pueden ser determinadas a partir de imágenes satelitales que posean datos térmicos.

El presente estudio tiene como objetivo determinar, a partir de una imagen Landsat 5TM la TS para los diferentes sectores de la zona nororiental de la península de Brunswick en un momento determinado. Para el cálculo del valor de cada píxel de la imagen se utilizaron algoritmos establecidos previamente. La comprobación del método se hizo comparando la valorización de los pixeles de la imagen con los datos de temperatura del aire registrados por la estación meteorológica Jorge Schythe. Como resultado se ha logrado aplicar el método de cálculo de variables climáticas a partir de imágenes satelitales en estas latitudes y se ha obtenido la cartografía que muestra la variabilidad de la temperatura superficial en la península de Brunswick.
\end{abstract}

Palabras clave: Teledetección, temperatura superficial, Landsat, Punta Arenas

\section{ABSTRACT}

Surface temperature (ST) has been historically recorded in meteorological stations at specific geographic locations. That data has been used later as a proxy for larger areas, despite knowing that ST changes accordingly to local factors, such as topography and latitude. More recently, small-scale variations on ST have been estimated by satellite images that reveal terrestrial ST. This study aimed to estimate ST from a Landsat 5TM image of the northeastern area of Brunswick Peninsula, using standard algorithms

Centro de Estudios el Cuaternario de Fuego-Patagonia y Antártica (CEQUA).

2 Laboratorio de Climatología, Instituto de la Patagonia, Universidad de Magallanes. 
for each pixel on the image. To test the method we compared the values estimated with those recorded at the Jorge Schythe meteorological station from the air temperature. We successfully applied here the method of climate variable calculation from satellite images and thus obtained a cartography showing the variability ST at Brunswick Peninsula.

Key words: Remote Sensing, Surface Temperature, Landsat, Punta Arenas

\section{INTRODUCCIÓN}

El uso de la tecnología espacial como herramienta complementaria en los estudios medioambientales, ha permitido un avance sustancial en la evaluación de la superficie terrestre y ha favorecido la eficiencia en la utilización de datos puntuales como los obtenidos por las estaciones meteorológicas. Además, permite reducir las salidas a terreno, especialmente a lugares remotos, limitando la toma de datos in situ a los necesarios para estandarizar los calculados a partir de imágenes satelitales.

La Teledetección o percepción remota se conoce como la ciencia de interpretar la superficie terrestre sin estar en contacto directo con ella. Tiene diversas aplicaciones en estudios del medio ambiente, tales como evaluación de coberturas, estado de la vegetación, agricultura de precisión, entre otras. La información de energía o radiancia emitida y reflejada por la superficie terrestre proporcionada por los satélites tales como Landsat, con un píxel de 30 metros de resolución espacial, ha sido una de las más utilizadas (Chuvieco 2002).

Los satélites Landsat TM (Thematic Mapper) 5 y Landsat 7 ETM + disponen de imágenes que cubren la región de Magallanes en diferentes estaciones del año, con una frecuencia o resolución temporal de 16 días. Aunque resulta difícil encontrar imágenes con cielos despejados y la mayor parte de las disponibles presentan un considerable porcentaje de nubosidad, algunas de ellas permiten una interpretación confiable de los datos.

La primera misión Landsat se realizó en 1972 con registros de imágenes hasta el año 1978, existiendo seis lanzamientos posteriores a éste, los que han ido mejorando en tecnología a través de los años. En la actualidad tres de estos satélites se mantienen operativos (Fig. 1) de los cuales sólo Landsat 5 es el que entrega datos completos; el Landsat 7, último en ser enviado al espacio, presentó una falla técnica en mayo del año 2003. (Landsat Program¹).

En la actualidad se cuenta con satélites de diferentes resoluciones y costos: Landsat, Ikonos, Aster, Modis, entre otros, los que entregan diferente tipo de información pero toda ella aplicable y complementaria a estudios del medio ambiente.

1 http://landsat.gsfc.nasa.gov/

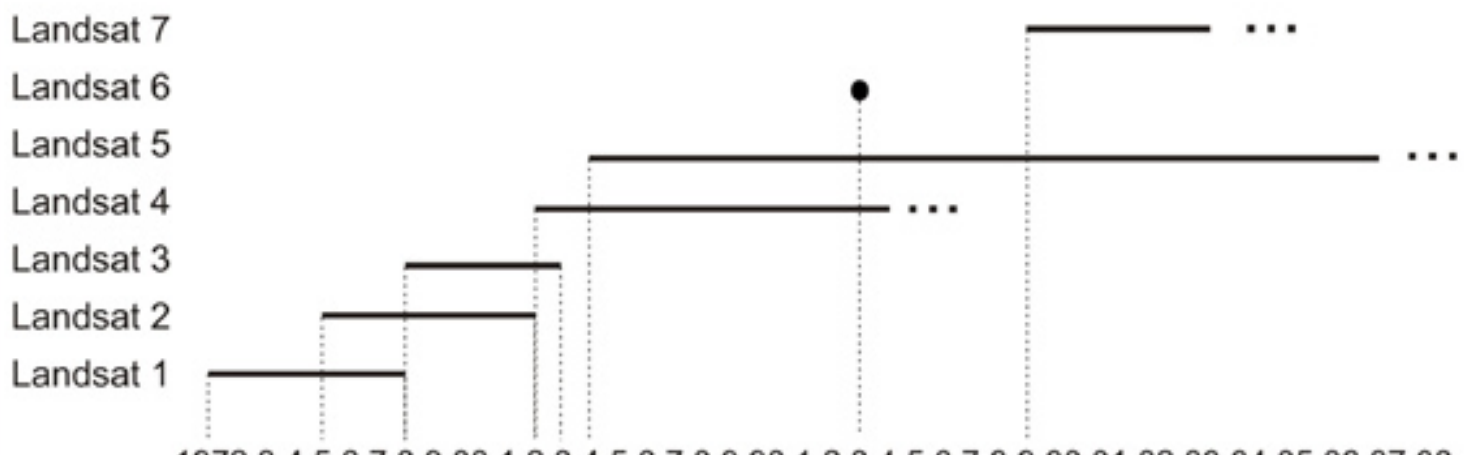

197234567898012345678990123456789000102030405060708

Fig. 1 Cobertura histórica de los satélites Landsat. Los (...) representan satélites operativos. 
TABLA 1. Longitud de onda por banda espectral para Landsat 5 (TM) y $7(E T M+)$, expresadas en $\mu \mathrm{m}$.

\begin{tabular}{|c|c|c|c|c|c|c|c|c|}
\hline Sensor & Banda 1 & Banda 2 & Banda 3 & Banda 4 & Banda 5 & Banda 6 & Banda 7 & Banda 8 \\
\hline TM & $0,45-0,52$ & $0,52-0,60$ & $0,63-0,69$ & $0,76-0,90$ & $1,55-1,75$ & $10,4-12,5$ & $2,08-2,35$ & No existe \\
\hline ETM+ & $0,45-0,52$ & $0,53-0,61$ & $0,63-0,69$ & $0,78-0,90$ & $1,55-1,75$ & $10,4-12,5$ & $2,09-2,35$ & $0,52-0,90$ \\
\hline Región & Visible azul & Visible verde & Visible rojo & $\begin{array}{c}\text { Infrarrojo } \\
\text { próximo }\end{array}$ & $\begin{array}{c}\text { Infrarrojo } \\
\text { lejano }\end{array}$ & $\begin{array}{c}\text { Térmico } \\
\text { lejano }\end{array}$ & $\begin{array}{c}\text { Térmico } \\
\text { próximo }\end{array}$ & Visible \\
\hline
\end{tabular}

Los satélites Landsat 5 y 7 cuentan con una resolución espacial ideal para cartografiar la superficie terrestre hasta una escala de 1:50.000 aproximadamente y su resolución espectral, permite discriminar en partes el espectro electromagnético, lo cual le da enormes ventajas a la hora de clasificar suelos o realizar aplicaciones específicas. En la Tabla 1 se muestra las bandas y rangos para Landsat 5 (TM) y 7(ETM+) (Landsat Program¹).

Por décadas, el clima ha sido un importante tema de estudio tanto a nivel científico como en muchos ámbitos de la vida del ser humano, ya que presenta una gran relevancia en la determinación de diferentes eventos y sistemas de su vida cotidiana. Dentro de las variables del clima, la temperatura es un resultado de la energía solar, por tanto es regida, principalmente, por el efecto de la latitud en el ángulo de inclinación en el que esta energía llega a la superficie terrestre, siendo mayor en el Ecuador y disminuyendo hacia los polos.

Esto último indica que existen diferentes niveles de TS, los que en la región de Magallanes, no han sido estudiados en detalle espacial continuo ni empleando metodologías modernas, sólo se cuenta con las contribuciones hechas por Jerez \& Arancibia (1972), INIA (1982) y Endlicher \& Santana (1988), las cuales se han enfocado a la clasificación del clima mediante datos de las estaciones climatológicas.

El uso de la información de Landsat en variables del clima también constituye una aplicación en este campo. Por ejemplo, basado en la banda térmica (Banda 6) de este satélite (Tabla 1) y utilizando algoritmos adecuados es posible estimar tanto el valor como la distribución de la temperatura en extensas áreas de la superficie terrestre. Por tanto, el objetivo de este trabajo es disponer de una primera aproximación al cálculo de la TS de la zona nororiental de la península de Brunswick, en la región de Magallanes, y mostrar la variabilidad térmica que existe en un momento determinado, lo que con técnicas convencionales podría ser caracterizada con el mismo valor.

\section{MATERIALES Y MÉTODOS}

Se seleccionó la imagen Landsat 5 TM del 10 de febrero de 1986, escena capturada a las 13:34 UTC, obtenida del Global Land Cover Facility (GLFC ${ }^{2}$, según sus siglas en inglés). La imagen incluye los tratamientos de ortorrectificación y georreferenciación en el DATUM WGS84 y para este trabajo se utilizó la proyección Universal Tranversa de Mercator (UTM). La banda térmica de este satélite (banda 6), que capta la superficie terrestre en un rango térmico del espectro electromagnético por cada píxel (Tabla 2 ), es capaz de discriminar la variación de TS en un momento determinado.

Para el procesamiento de datos obtenidos por la banda térmica del satélite se utilizó el software Erdas Imagine, que permite aplicar algoritmos de corrección que disminuyen la interferencia ocasionada por la atmósfera, y, en consecuencia los errores que esto pudiera causar. Posteriormente se utilizó la extensión Modeler del mismo software para introducir las fórmulas e imagen respectivamente, con las cuales se obtuvieron los valores de radiancia y temperaturas.

El proceso de cálculo de las temperaturas comienza con los niveles digitales (ND) de la banda térmica, detectados por el sensor satelital. Debido a que se trata de un sensor de 8 bit, el píxel puede

2 http://glcfapp.umiacs.umd.edu:8080/esdi/index.jsp

TABLA 2. Características de la banda 6 de Landsat 5

\begin{tabular}{|c|c|c|c|c|}
\hline Satélite & Numero de Banda & Rango del espectro $(\mu \mathrm{m})$ & $\begin{array}{c}\text { Resolución } \\
\text { espacial }(\mathrm{m})\end{array}$ & $\begin{array}{c}\text { Escala Radiométrica } \\
\left(\mathrm{W} / \mathrm{m}^{2} * \mathrm{sr}^{*} \mu \mathrm{m}\right)\end{array}$ \\
\hline Landsat 5 TM & 6 & 10,45 a 12,42 & 120 & $1,238-15,300$ \\
\hline
\end{tabular}


tomar un valor entre 0 y 255 niveles de grises. Al igual que en el rango óptico del espectro, esos valores codifican un valor de radiancia correspondiente al infrarrojo térmico (Chuvieco 2002). La obtención de las temperaturas de la superficie terrestre a partir de la banda térmica de Landsat 5, conlleva la aplicación de una serie de fórmulas para la calibración adecuada de cada tipo de imagen, dependiendo del sensor (Barsi et al. 2003, Xian \& Crane 2006, Rigo et al. 2006).

La radiancia espectral emitida se calculó mediante la fórmula (1) (Tomada de Wukelic et al. 1989 y Xian \& Crane 2006), donde los factores de calibración Gain (G) y Bias (B) se obtuvieron de la metadata de la imagen satelital

$$
L=G \times N D+B
$$

Donde:

$\mathrm{L}=$ es el valor de píxel expresado en radiancia

ND = es el nivel digital del píxel

$\mathrm{G}=$ es el factor de gain y en este caso toma un valor de 0.551582 .

$\mathrm{B}=$ es el factor de bias y en este caso toma un valor de 1.2377996 .

La temperatura de brillo, emitida por la superficie terrestre, se obtuvo transformando la banda TM (6) del infrarrojo térmico mediante la inversión de la ecuación de Planck (fórmula (2)) (Wukelic et al. 1989, Riaño et al. 2000, Xian \& Crane 2006) la cual describe la emisión de energía de un cuerpo en función de su longitud de onda a una temperatura dada. Las constantes utilizadas, son específicas para Landsat TM5.

$$
T=\frac{K_{1}}{\ln \left(\frac{K_{2}}{L}+1\right)}
$$

Donde:

$\mathrm{T}=$ es la temperatura de brillo superficial

$\mathrm{L}=$ es el valor de radiancia

$\mathrm{K}_{1}=$ es la constante 607.76

$\mathrm{K}_{2}=$ es la constante 1260.56

Con la finalidad de comprobar los cálculos obtenidos a partir de Landsat 5TM se compara- ron los datos estimados para la valorización de los píxeles con datos climáticos registrados por la estación Jorge Schythe, georreferenciada mediante GPS Garmin etrex vista para posicionarla en el sector de la imagen y el termograma correspondiente al día y a la hora en que esta se capturó. En forma adicional, se compararon los valores de TS estimados para el estrecho de Magallanes con los promedio de la temperatura superficial del agua de mar en el estrecho informados en los trabajos de de Andrade (1991) y Valdenegro \& Silva (2003) que son los más completos en cuanto a caracterización oceanográficas de la Región de Magallanes.

\section{Área de estudio}

La península de Brunswick, ubicada en los $52^{\circ} 17^{\prime}$ y $53^{\circ} 35^{\prime}$ de latitud sur y los $70^{\circ} 23^{\prime}$ y $71^{\circ} 33^{\prime}$ de longitud oeste, en el sector centro-oriental de la región de Magallanes, incluye a la ciudad de Punta Arenas en su parte media. Tiene una superficie total de $5800 \mathrm{~km}^{2}$ aproximadamente. El gradiente térmico en el área, aumenta en dirección sur a norte, comenzando con un ambiente montañoso al sur, hasta llegar a la estepa por el norte (Fig. 2)

\section{RESULTADOS}

Se presentan los valores de temperatura superficial para la zona noreste de la península de Brunswick, estimados mediante algoritmos de transformación de datos de niveles digitales obtenidos de una imagen satelital Landsat 5 TM. Para disminuir el efecto de disgregación de los datos, los valores se agruparon en rangos de temperatura representados por diferentes colores (Fig. 3).

La comprobación de los datos entregados por la imagen satelital del día 10 de febrero de 1986 a las 13:34 hrs. UTC, se hizo comparando el registro de la temperatura del aire para la ciudad de Punta Arenas en ese día y horario, para la cual el termograma registró $9,0^{\circ}$ Celsius (C) y el valor de TS estimado de la imagen satelital que fue de 9,7 $7^{\circ}$; el segundo punto de comparación fue el valor promedio de la temperatura superficial del agua del mar en el estrecho de Magallanes, para la cual Andrade (1991) informa valores del orden de los $3^{\circ}$ a $11^{\circ} \mathrm{C}$ y Valdenegro \& Silva (2003) 


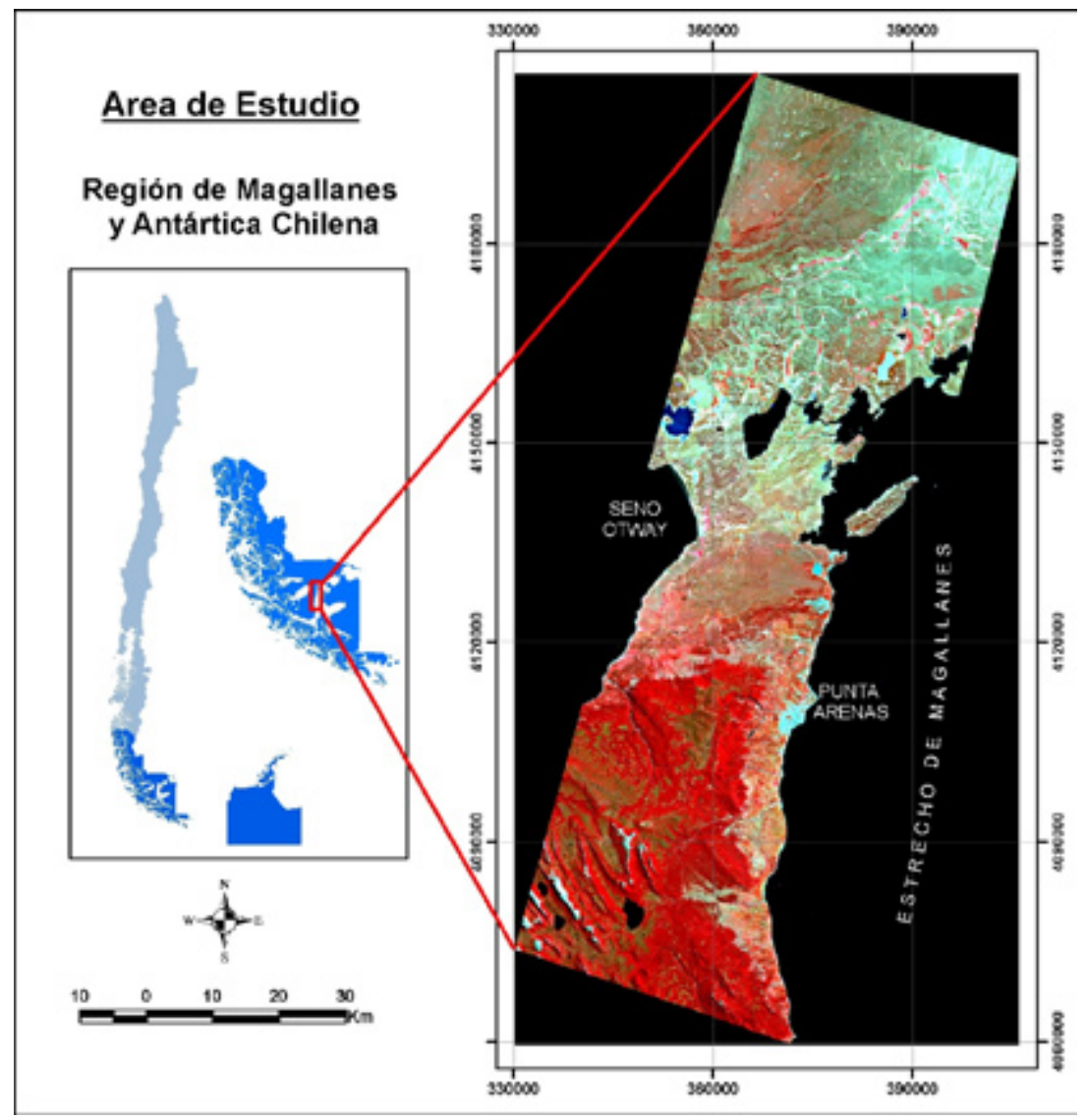

Fig. 2. Área de estudio. Península de Brunswick, Imagen Landsat 5 TM, 10 de febrero, 1986 falso color convencional (432 RGB).

caracterizan a la zona central del estrecho de Magallanes con una temperatura promedio de $7,0^{\circ}$ C. La TS en el estrecho de Magallanes, estimada a partir de la imagen satelital, en este estudio, fue de $3,7^{\circ}$ a $4^{\circ} \mathrm{C}$.

La imagen utilizada en este estudio alcanza una extensión territorial no mayor a $120 \mathrm{~km}$ de largo en los cuales la variabilidad de la TS va desde los $24^{\circ} \mathrm{C}$ en el extremo norte de la península de Brunswick hasta los $-4^{\circ} \mathrm{C}$ en la parte sur (Fig. 3)

La imagen de verano empleada muestra con claridad la variabilidad de las temperaturas en la zona de estepa, las que se distribuyen, mayoritariamente, entre los $24^{\circ}$ y $14^{\circ} \mathrm{C}$. La zona trasandina está caracterizada por temperaturas que varían entre los $14^{\circ}$ y $6^{\circ} \mathrm{C}$ y en la zona sur oriental de la península, que corresponde al sector montañoso, la temperatura varía entre los $6^{\circ} \mathrm{y}-4^{\circ} \mathrm{C}$.

\section{DISCUSIÓN}

En cuanto a las variables de comparación utilizados, cabe señalar que la temperatura del aire y la TS son parámetros meteorológicos diferentes, pero estrechamente relacionados, por lo cual, para efectos de comparación, se espera que los valores de ambas sean muy cercanos. Es así como la temperatura del aire registrada por la estación Jorge Schythe el 10 de febrero de 1986 a las 13:34 hrs. fue de $9,0^{\circ} \mathrm{C}$ y para el mismo día y hora la TS estimada por la imagen Landsat fue de $9,7^{\circ} \mathrm{C}$. En relación a esto, se debe considerar que la imagen Landsat utilizada corresponde al verano austral, estación en la cual la radiación solar es máxima en el hemisferio sur y la absorción de ella por parte de la superficie terrestre es mayor a la temperatura que alcanza el aire, lo que es coincidente con la comparación obtenida. Por otra parte la diferencia de 0,7 


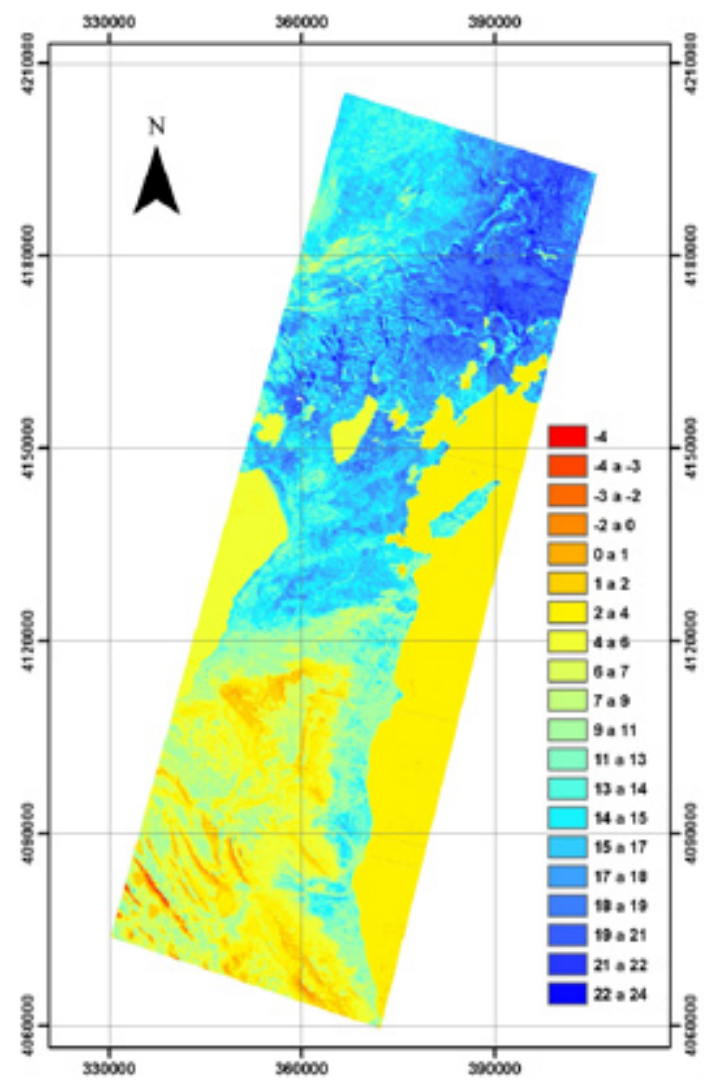

Fig. 3. Distribución de las TS en el sector nororiental de península de Brunswick, Los rangos de temperatura $\left({ }^{\circ} \mathrm{C}\right)$ están representadas en colores.

décimas de grado entre la TS y la del aire pueden ser explicadas porque esta última, por convención universal, es tomada a la sombra.

En relación al segundo punto de comparación, se puede precisar que la temperatura superficial del agua de mar, a lo largo del estrecho de Magallanes, se mantiene homogénea entre 3,7 y $4^{\circ} \mathrm{C}$ según los valores estimados a partir de la imagen satelital, a diferencia de los fluctuantes valores que toma este parámetro, en los diferentes puntos de medición en el estrecho de Magallanes presentados en los trabajos de Andrade (1991) y Valdenegro \& Silva (2003), los que se encuentran entre el rango de los 3 y $11^{\circ} \mathrm{C}$. Este amplio intervalo se debe, principalmente a que estos registros corresponden al promedio de valores tomados a diferentes horas del día, durante varios días y en diferentes lugares a través del estrecho de Magallanes. Por lo mismo, Andrade (1991) concluye que la descripción general entregada en su estudio podría mejorarse usando imágenes de satélite para las diferentes estaciones del año.

El método de cálculo de la TS a partir de imágenes satelitales es aplicable en esta parte del hemisferio y confiable para estimar la variación de la TS en la península de Bruswick, ya que los valores de TS estimados de la imagen satelital son cercanos y están dentro del rango esperado de los puntos de comparación.

Según la clasificación de Köppen adaptada por Pisano (1977) y Zamora \& Santana (1979), en el sector estudiado se encuentran dos tipos de clima: Clima de Estepa frío y Clima Trasandino con Degeneración Esteparia, para los cuales hemos estimado 28 grados de variación en la TS. En la zona norte de la península, que es un sector de estepa con una altitud máxima de $300 \mathrm{~m}$ (datos obtenidos a partir de modelos digitales de terreno SRTM $^{3}$ Shuttle Radar Topographic Mission, según sus siglas en inglés, disponibles en el $\mathrm{GLFC}^{2}$ ) se encuentran los mayores valores de TS $\left(24^{\circ} \mathrm{C}\right)$ y en la zona sur oriental de la península de Brunswick, que es un sector montañoso con altitudes máximas de 900 m (SRTM ${ }^{3}$, disponibles en el $\left.\mathrm{GLFC}^{2}\right)$ los valores de temperatura en la superficie terrestre llegaron a $-4^{\circ} \mathrm{C}$. Debemos recordar que los valores de TS están influenciados en general por la latitud y específicamente por el tipo de cobertura terrestre la que, a su vez, está determinada por las condiciones ambientales en el sector, cuya distribución depende de la morfología y circulación climática presentes en la región (Endlicher \& Santana 1988). En el sector estudiado la TS está determinada, principalmente, por factores locales como la cobertura, la altitud y la topografía del terreno, la que al ser más accidentada, modifica la corriente principal de los vientos y en consecuencia permite una rápida disminución de la temperatura, como lo encontrado en el sector montañoso de la península.

Entre las muchas aplicaciones de las imágenes satelitales Landsat se pueden destacar los trabajos de Recondo \& Pérez-Morandeira (2002), quienes estimaron la temperatura del aire a partir de la temperatura de brillo del suelo o temperatura superficial, aún cuando para ello se debe contar con una gran cantidad de estaciones meteorológicas que permitan calcular la diferencia que hay entre

\footnotetext{
3 http://srtm.usgs.gov/
} 
la temperatura a nivel del satélite y la temperatura del aire. Sánchez (2002), por su parte, destaca que la posibilidad de establecer una relación entre los datos continuos entregados por una imagen satelital y los obtenidos de manera convencional, resulta una herramienta eficaz para obtener valores de variables climáticas que complementan los registros de las estaciones en sectores donde éstas no están presentes. Los resultados de este estudio son útiles al momento de planear la ubicación de más estaciones meteorológicas que permitan tener una base de datos de puntos de comprobación para, a futuro, poder determinar los factores climáticos de zonas remotas de nuestra región, donde definitivamente no sea posible instalar estaciones climáticas y/o dar seguimiento al registro de los datos, para estimar otros parámetros meteorológicos a partir de la TS o mediante el cálculo con otras bandas del satélite con el objeto de complementar estas parámetros en investigaciones multi e interdiciplinarias.

En un futuro próximo se contará con una antena de recepción satelital en la región de Magallanes, con la que se tendrá una serie continua de imágenes, las que a la fecha son de acceso limitado. En forma tangible, el contar con estas imágenes, abre una gama de posibilidades de estudios innovadores que permitirán hacer un catastro de variables climáticas las cuales, mediante correcciones sistemáticas y algoritmos, permitirán complementar los estudios temporales y comparativos aplicadas a la cartografía de modelos, (como los de incendios de áreas) o complementar los estudios florísticos y faunísticos de la zona y en el propio ordenamiento territorial.

\section{CONCLUSIONES}

Se ha logrado caracterizar la variabilidad de la TS en el sector nororiental de la península de Brunswick, en un momento determinado, empleando una imagen Landsat 5 TM.

Este trabajo es uno de los primeros que utiliza una imagen de satélite para calcular la TS en un sector de la región de Magallanes, comprobando su aplicación con registros convencionales de variables meteorológicas del mismo día y hora en que fue capturada la imagen.

La banda térmica de la imagen Landsat 5 TM entrega información adecuada para el cálculo de valores de TS y su distribución espacial en un momento determinado. Por tanto se recomienda su aplicación para la obtención de datos climáticos, principalmente en zonas apartadas carentes de estaciones meteorológicas.

Se plantea la necesidad de instalar más estaciones meteorológicas en la región de Magallanes que permitan formar una base de datos de referencia para estandarizar los cálculos de variables climáticas a partir de las diferentes bandas del sensor satelital, para hacer caracterizaciones climáticas de zonas remotas de la región.

Este método es una herramienta de obtención de temperaturas útil para complementar estudios biológicos multidisciplinarios.

Actualmente el satélite Landsat 5 TM sigue registrando datos espaciales, lo cual hace posible continuar con este tipo de estudios, sin embargo, se deben considerar todos aquellos satélites que posean una o más bandas térmicas como potenciales instrumentos de monitoreo climático, por ejemplo Modis que entrega un producto de TS y Meris que también tiene bandas térmicas.

\section{AGRADECIMIENTOS}

Queremos reconocer y agradecer el esfuerzo del Laboratorio de Climatología del Instituto de la Patagonia por el continuo registro de los datos climáticos de la Estación Jorge Schythe a su cargo y a los observadores de mencionada estación Sr. José Bárcenas (†) y Sr. Rodrigo Ojeda, datos sin los cuales este trabajo no habría sido posible. Agradecemos la hospitalidad del Laboratorio de Climatología al permitir espacio físico para el desarrollo del trabajo de los autores. Finalmente agradecemos a la fundación CEQUA por las facilidades prestadas para la realización de este estudio.

\section{LITERATURA CITADA}

Andrade S. 1991. Geomorfología costera y antecedentes oceanográficos físicos de la región

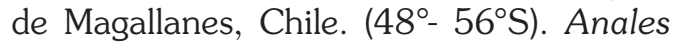
Instituto Patagonia. Serie Cs. Nat. (Chile) 20 (1):135-151.

Barsi J., J. Schott, F. Palluconi, D. Helder, S. Hook, B. Markham, G. Chander \& E. O'Donnell 2003. Landsat TM and ETM+ thermal band 
calibration. Canadian Journal of Remote Sensing 29:141-153

Chuvieco E. 2002. Teledetección Ambiental - La observación de la tierra desde el espacio. Editorial Ariel S.A. Primera edición, Madrid, España.

Endlicher W. \& A. Santana 1988. El clima del sur de la Patagonia y sus aspectos ecológicos. Un siglo de mediciones climatológicas en Punta Arenas. Anales Instituto Patagonia (Chile) 18:57-86.

INIA. 1982. Distritos agroclimáticos: cartas de isolíneas climáticas. Estudio plan de desarrollo tecnológico agropecuario, Vol. 7 unidad de trabajo $n^{\circ} 3$, Estación Kampenaike, Punta Arenas, Chile.

Jerez M. \& M. Arancibia 1972. Trazado de isoyetas del sector centro oriental de la provincia de Magallanes. Instituto de la Patagonia. Monografía $\mathrm{N}^{\circ} 4$. Punta Arenas, Chile.

Pisano E. 1977. Fitogeografía de Fuego - Patagonia chilena. I.- Comunidades vegetales entre las latitudes $52^{\circ}$ y $56^{\circ}$. Anales Instituto Patagonia (Chile) 8:121-250

Recondo C. \& C. Pérez - Morandeira 2002. Obtención de la temperatura del aire de Asturias a partir de la temperatura de superficie calculada con imágenes NOAA - AVHRR. Revista de Teledetección 17:5-12.

Riaño D., J. Salas \& E. Chuvieco 2000. Corrección Atmosférica y Topográfica, Información Multitemporal y Auxiliar Aplicadas a la Cartografía de Modelos de Combustibles con Imágenes Landsat-TM. pp. 222-239. En: Tecnologías Geográficas para el Desarrollo Sostenible,. Aguado, I. y Gómez. M. ED., Departamento de Geografía, Universidad de Alcalá.

Rigo G., E. Parlow \& D. Oesch 2006. Validation of satellite observed thermal emission with in-situ measurements over an urban surface. Remote Sensing of Environment 104: 201-210.

Sánchez M. 2002. Modelos y aproximaciones para la estimación de la evapotranspiración con información satelital. Revista de Geografía Norte Grande 29: 107-120

Valdenegro, A. \& N. Silva 2003. Caracterización oceanográfica física y química de la zona de canales y fiordos australes de Chile entre el estrecho de Magallanes y Cabo de Hornos (CIMAR 3 FIORDOS). Ciencia y Tecnología del Mar 26 (2): 19-60.

Wukelic, G., D. Gibbons, L. Martucci \& H. Foote 1989. Radiometric calibration of Landsat Thermatic Mapper thermal band. Remote Sensing of Environment 28:339-347.

Xian, G. \& M. Crane 2006. An analysis of urban thermal Characteristics and associated land cover in Tampa Bay and Las Vegas using Landsat satellite data. Remote Sensing of Environment 104 (2006) 147-156

Zamora, E. \& A. Santana 1979. Características climáticas de la costa occidental de la Patagonia entre las latitudes $35^{\circ} 30^{\prime} S$ y $56^{\circ} 30^{\prime}$ S. Anales Instituto Patagonia (Chile) 10: 109-14. 